des geringsten Widerstandes herausgepresst würde, das hat $\mathrm{H}$ of meister schlagend bewiesen. Den Beweis liefert die leicht zu bestätigende Thatsache, dass erst die Ausstülpungen sich bilden und dann nachträglich die Körnchen in sie hineinströmen. Die Körnchen kommen dabei um so später in Bewegung, je weiter sie vom Ziel der Strömung entfernt sind: die Körnchenbewegung greift rückwärts uın sich. In unserm Falle also würde, wenn man überhaupt von Contraction sprechen will, die Bildung jener sphärisch begrenzten Protoplasmaausstülpungen nicht auf einer Contraction entfernterer Theile des Protoplasma, sondern auf Contraction des Protoplasma der ausgestülpten Partie selbst beruhen. Die Kräfte aber, welche diese Contraction bewirken, würden - dafür sprechen unsere obigen Beobachtungen - ganz dieselben sein können, wie die, welche jeden nicht kugligen freien Flüssigkeitstropfen kuglig zu machen streben.

\title{
Die Grenzen der Schmeckbarkeit von Chlornatrium in wässriger Lösung.
}

\author{
Von \\ Dr. Camerer, \\ pract. Arzt in Gerstetten, Würtemberg.
}

Neben dem wissenschaftlichen Interesse, welches die Leistungen auch der minder wichtigen Sinne erregen, ist der Geschmackssinn für den Arzt bemerkenswerth und das Studium seiner Anomalien dürfte, nach Feststellung der normalen Verhältnisse, nicht ohne praktischen Werth sein.

Versuche äber die Feinheit des Geschmacksinns hat schon vor langer Zeit Valentin mit 6 verschiedenen Stoffen angestellt (Lehrb. der Physiologie Band 2, p. 274). Seine Angaben über Chlornatrium sind folgende; 
Die Grenzen der Schmeckbarkeit von Chlornatrium in wässriger Lösung. 323

\begin{tabular}{c|c|c|c}
\hline $\begin{array}{c}\text { In 10,000 Thei- } \\
\text { len Wasser ent- } \\
\text { haltene Salz- } \\
\text { menge }\end{array}$ & $\begin{array}{c}\text { Verschlucktes } \\
\text { Quantum } \\
\text { in ccm. }\end{array}$ & $\begin{array}{c}\text { Absolute Salz- } \\
\text { menge in } \\
\text { Milligramm. }\end{array}$ & \\
\hline 470 & 0,1 & 5 & sehr deutlich salzig. \\
47 & 0,5 & 2 & Geschm. schwach. \\
47 & 1,5 & 6 & Zieml. deutl. salzig. \\
47 & 3,0 & 12 & schwach aber deut1. \\
24 & 8,0 & 19 & kein deatl. Geschm. \\
24 & 12,0 & 29 & äus. schwach salz.
\end{tabular}

Die folgenden, nach der Methode der richtigen und falschen Fälle angestellten Versuche sollen die Grenzer der Schmeckbarkeit für Chlornatrium, den Einfluss der Tageszeit und der Temperatur der Lösung hierauf feststellen.

1. Technik und Normalbedingungen der Versuche.

Das verwandte reine Steinsalz aus der Saline Friedrichshall ist reines wasserfreies Chlornatrium; das Lösungswasser ist, so lange nichts weiteres bemerkt wird, durch Schneeschmelzen erhalten. Aus gesättigter Salzlösung, zu deren sichern Herstellung Wasser und Salz etwa 2 Monate gemischt bleiben muss, wurden je $5000 \mathrm{ccm}$. der hundertfachen Verdünnung im Vorrath und aus dieser kurz vor dem Versuch die zu demselben bestimmten „Lösungen“ bereitet. Der letzteren waren es 5; eingeschaltet wurden 1-2 Proben reines Wasser, um die Versuchsperson durch Vexirversuche zu prüfen. Aus kleinen Trinkgläschen wurden $30 \mathrm{ccm}$. in den Mund genommen; die Zunge und Lösung möglichst ruhig gehalten, nach erfolgter Empfindung wurde die Lösung wieder ausgespieen. Die hintern Partien der Mundhöhle kommen daher nicht in Betracht. Von der Folge der Lösungen vom concentrirten zum schwachen und umgekehrt, wird später die Rede sein. Die Temperatur der Lösung betrug für gewöhnlich $13-15^{\circ}$ Celsius.

Die Versuchszeit dauerte an einem Tag $1 \frac{1 / 2}{2}-2$ Stunden; zwischen jedem Einzelversuch liegen 2 Minuten. Die 6, resp. 7 Versuche mit den Lösungen und dem Vexirwasser wurden ohne weitere Unterbrechung gemacht - ich nenne diess im Folgenden Versuchsreihe - sodann 5 Minnten pausirt. Zwischen den Versuchen und der vorhergehenden Mahlzeit liegen 2 Stunden. Alle Versuche sind gleichzeitig von mir und meiner Frau, 
beide gegenwärtig 27 Jahre alt, in der Art gemacht, dass das Resultat während des Versuchstags dem Betreffenden unbekannt blieb.

Ich selbst bin ein mässiger Tabakraucher. - Manche andere Verhältnisse konnte ich der Umstände halger nicht in ganz befriedigender Weise reguliren; namentlich erstrecken sich die Versuche über einen grossen Zeitraum vom November 67 bis zum März 69, da meine Landpraxis der schnellen Ansammlung vieler Erfahrungen ihre gemessene Grenze setzte.

In den folgenden Tabellen ist die Anzahl der Entscheidungen „richtig“ enthalten; die Entscheidung „unbestimmt" ist halb zu richtig und halb zu falsch gezählt. Um die hierdurch auftretenden Decimale zu vermeiden, erscheint die Anzahl der Entscheidungen verdoppelt. Meine Urtheile sind in der Rubrik I, die meiner Frau in der Rubrik II enthalten.

Der Salzgehalt der Lösungen in Grammen ist folgender :

Tabelle a.

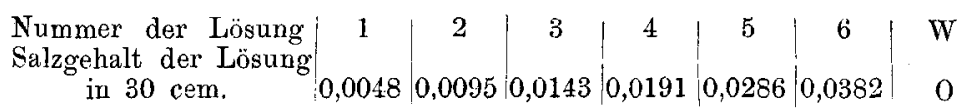

2. Versuche mit geänderter Tageszeit.

Tabelle $b$.

\begin{tabular}{|c|c|c|c|c|c|c|c|c|}
\hline Numme & Cösung & 1 & $2 !$ & 3 & \pm & 5 & & \\
\hline Urtheil & $\begin{array}{r}\text { II } \\
\mathrm{I}+\mathrm{II}\end{array}$ & $\begin{array}{c}12 \\
2 \\
14\end{array}$ & $\begin{array}{l}34 \\
62 \\
96\end{array}$ & $\begin{array}{l}69 \\
84 \\
153\end{array}$ & $\begin{array}{r}87 \\
90 \\
177\end{array}$ & $\begin{array}{r}99 \\
100 \\
199\end{array}$ & $\mid$\begin{tabular}{|c|}
94 \\
95 \\
189
\end{tabular} & 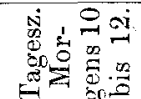 \\
\hline Urtheil & $\begin{array}{r}\mathrm{I} \\
\mathrm{II} \\
\mathrm{I}+\mathrm{II}\end{array}$ & $\begin{array}{l}15 \\
6 \\
21\end{array}$ & $\begin{array}{l}42 \\
57 \\
99\end{array}$ & $\begin{array}{c}84 \\
166\end{array}$ & $\begin{array}{c}96 \\
189\end{array}$ & \begin{tabular}{|l|}
98 \\
98 \\
196
\end{tabular} & \begin{tabular}{|l|}
98 \\
95 \\
193
\end{tabular} & 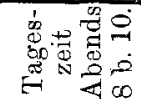 \\
\hline
\end{tabular}

Absolute Zahl

d. Versuchsreihen je 50, d. Einzelversuche je 30 ).

Diese Versuche sind vom 25. November bis 11 . Dezember 67 gemacht und zwar bei der Hälfte der Fälle Morgens und Abends an demselben Tag. - Falsche Urtheile beim Wasser kommen so selten vor, dass den positiven Urtheilen sogar bei der stärksten Verdünnung wenigstens für I volle Beweiskraft zu schenken ist. Für II zeigt sich kein Unterschied, für I ein unerheblicher Vortheil zu Gunsten des Abends, welcher übrigens bei Fraktionirung der Versuchsreihen als überall vorkommend und nicht von einzelnen begünstigten Reihen herrührend sich erweist. 
Die Gränzen der Schmeckbarkeit von Chlornatrium in wässriger Lösung. 325

3. Versuche mit geänderter Temperatur.

Tabelle c.

\begin{tabular}{|c|c|c|c|c|c|c|c|c|c|c|}
\hline \multicolumn{2}{|c|}{ Nummer d. Lösung } & 2 & 3 & 4 & 5 & 6 & $\mathrm{~W}$ & W & $\begin{array}{l}\text { Tempera- } \\
\text { tur in }{ }^{0} \\
\text { Celsius } \\
\end{array}$ & \multirow{6}{*}{$\begin{array}{l}\text { Absolute Zahl der } \\
\text { Versuchsreihen je } 50 . \\
\text { Absolute Zahl der } \\
\text { Einzelversuche je } 350 .\end{array}$} \\
\hline Urtheil & $\begin{array}{r}I \\
I I \\
I+I I\end{array}$ & \begin{tabular}{l|l}
23 \\
14 \\
37
\end{tabular} & $\mid \begin{array}{l}45 \\
41 \\
86\end{array}$ & $\mid \begin{array}{c}68 \\
80 \\
148\end{array}$ & \begin{tabular}{|c|}
80 \\
93 \\
173
\end{tabular} & $\left|\begin{array}{c}94 \\
95 \\
189\end{array}\right|$ & $\left|\begin{array}{r}98 \\
100 \\
198\end{array}\right|$ & $\begin{array}{r}99 \\
95 \\
194\end{array}$ & 5 & \\
\hline Urtheil & $\begin{array}{r}\mathrm{I} \\
\mathrm{II} \\
\mathrm{I}+\mathrm{II}\end{array}$ & $\begin{array}{l}36 \\
21 \\
57 \\
\end{array}$ & \begin{tabular}{|l|}
45 \\
48 \\
93
\end{tabular} & $\begin{array}{r}71 \\
88 \\
159 \\
\end{array}$ & $\begin{array}{r}97 \\
94 \\
191\end{array}$ & $\begin{array}{l}100 \\
100 \\
200\end{array}$ & $\begin{array}{l}100 \\
100 \\
200\end{array}$ & $\begin{array}{l}100 \\
100 \\
200\end{array}$ & 10 & \\
\hline Urtheil & $\begin{array}{r}\mathrm{I} \\
\mathrm{II} \\
\mathrm{I}+\mathrm{II}\end{array}$ & \begin{tabular}{|c|}
12 \\
7 \\
19 \\
\end{tabular} & \begin{tabular}{|l|}
41 \\
68 \\
109 \\
\end{tabular} & $\left|\begin{array}{c}63 \\
93 \\
155\end{array}\right|$ & $\left|\begin{array}{c}96 \\
98 \\
194\end{array}\right|$ & \begin{tabular}{|c|}
97 \\
98 \\
195
\end{tabular} & $\begin{array}{c}100 \\
97 \\
197\end{array}$ & $\left|\begin{array}{r}93 \\
99 \\
192\end{array}\right|$ & 35 & \\
\hline Urtheil & $\begin{array}{r}I \\
I I \\
I+I I\end{array}$ & $\begin{array}{l}13 \\
11 \\
24\end{array}$ & $\mid \begin{array}{l}34 \\
50 \\
84\end{array}$ & \begin{tabular}{|c|}
60 \\
78 \\
138
\end{tabular} & $\left|\begin{array}{c}86 \\
99 \\
185\end{array}\right|$ & $\left|\begin{array}{c}89 \\
100 \\
189\end{array}\right|$ & \begin{tabular}{|c|}
98 \\
100 \\
198
\end{tabular} & $\left|\begin{array}{c}93 \\
100 \\
193\end{array}\right|$ & 50 & \\
\hline Urtheil & $\begin{array}{r}I \\
I I \\
I+I I\end{array}$ & $\mid \begin{array}{c}16 \\
6 \\
22\end{array}$ & $\left|\begin{array}{l}27 \\
24 \\
51\end{array}\right|$ & $\left|\begin{array}{l}33 \\
28 \\
61\end{array}\right|$ & $\left|\begin{array}{c}56 \\
72 \\
128\end{array}\right|$ & $\begin{array}{r}86 \\
99 \\
185\end{array}$ & $\left|\begin{array}{c}97 \\
99 \\
196\end{array}\right|$ & $\left|\begin{array}{c}94 \\
100 \\
194\end{array}\right|$ & 60 & \\
\hline Urtheil & $\begin{array}{r}\mathrm{I} \\
\mathrm{II} \\
\mathrm{I}+\mathrm{II}\end{array}$ & $\begin{array}{l}1 \\
7 \\
8\end{array}$ & $\begin{array}{c}8 \\
21 \\
29\end{array}$ & $\left|\begin{array}{l}13 \\
24 \\
37\end{array}\right|$ & $\left|\begin{array}{l}24 \\
26 \\
50\end{array}\right|$ & $\mid \begin{array}{l}24 \\
26 \\
50\end{array}$ & $\left|\begin{array}{l|}23 \\
26 \\
49\end{array}\right|$ & $\left|\begin{array}{l}24 \\
26 \\
50\end{array}\right|$ & 20 & \multirow{3}{*}{$\begin{array}{l}\text { Absolute Zahl der } \\
\text { Versuchsreihen je } \\
12-13 \text {, der Einzel- } \\
\text { vers. je } 84-91 \text {. }\end{array}$} \\
\hline Urtheil & $\begin{array}{r}I \\
I I \\
I+I I \\
\end{array}$ & $\begin{array}{l}5 \\
2 \\
7 \\
\end{array}$ & \begin{tabular}{|l|}
5 \\
1 \\
6 \\
\end{tabular} & $\mid \begin{array}{l}11 \\
12 \\
23\end{array}$ & $\begin{array}{l}24 \\
22 \\
46\end{array}$ & $\left|\begin{array}{l}26 \\
20 \\
49\end{array}\right|$ & $\begin{array}{l}24 \\
24 \\
48\end{array}$ & {$\left[\begin{array}{l}24 \\
24 \\
48\end{array}\right]$} & 30 & \\
\hline Urtheil & $\begin{array}{r}I \\
I I \\
I+I I\end{array}$ & $\begin{array}{r}6 \\
8 \\
14\end{array}$ & $\begin{array}{l}16 \\
16 \\
32\end{array}$ & $\begin{array}{l}19 \\
20 \\
39\end{array}$ & $\begin{array}{l}25 \\
24 \\
49\end{array}$ & $\begin{array}{l}26 \\
21 \\
50\end{array}$ & $\begin{array}{l}26 \\
24 \\
50\end{array}$ & $\left|\begin{array}{l}25 \\
24 \\
49\end{array}\right|$ & 40 & \\
\hline
\end{tabular}

Diese Versuche sind vom Februar 68 bis zum März 69 gemacht und es sind auf jede der 5 Haupttemperaturen 8 Versuchstage verwendet. Sie sind auf einen Zetraum von wenigstens $11 / 2$ Monaten vertheilt, um der in kurzer Zeit merkbar schwankenden Empfindlichkeit (bei der' geringen Anzahl von Versuchstagen) keinen allzugrossen Einfluss einzuräumen.

Die Temperaturen $5^{\circ}$ und $60^{\circ}$ erregen bereits in geringem Grade Schmerz. Für I ist entschieden T $10 \mathrm{im}$ Vortheil, dann folgt $\mathrm{T} 5$, dem $\mathrm{T} 35$ wenig nachsteht, ebenso ist der Unterschied zwischen $\mathrm{T} 35$ und $\mathrm{T} 50$ unbedeutend, sehr bedeutend der zwischen $\mathrm{T}$ 50 and $\mathrm{T} 60$. Für II ist zwischen $\mathrm{T} 10$ und $\mathrm{T} 35$ kein Unterschied, ebenso sind T 5 und T 50 annähernd gleich, 60 sehr viel schlechter.

$\mathrm{Zu}$ diesen Versuchen musste jch - bei Mangel an Regen oder Flusswasser - gewöhnliches Quellwasser benutzen, welches kohlensauren Kalk und freie Kohlensäure in bemerkbarer Menge enthält; 
daher ist die Vergleichung mit den Versuchen unter 2) erschwert; allein mit ziemlicher Sicherheit wird sich sagen lassen, dass zwischen 'T 10 und 20 am Besten geschmeckt wird und bis zum beginnenden Schmerzgefühl die Leistung des Sinnes sich wenig ändert - was mit den Erfahrungen des täglichen Lebens stimmt. Die Kälte ist insofern begünstigt, als sie in geringem Grade zuerst an den Zähnen, nicht unmittelbar an den Weichtheilen schmerzt. Für ganz genaue Resultate sind meine Versuche zu wenig zahlreich; eine bedeutende Fehlerquelle liegt darin, dass bei diesen Temperaturversuchen das Urtheil sehr rasch gewonnen werden muss, weil sich sonst die Temperatur der Lösung im Munde zu stark ändert. Man hat nicht selten, aber nicht entschieden genug, um solche Fälle auszuschliessen, erst beim Ausspeien die Empfindung des Salzigen. Die wenigen folgenden Versuche enthalten in erster Reihe die Temperatur der gesammten Lösung; in zweiter Reihe die Temperatur der $30 \mathrm{ccm}$. unmittelbar nach dem Einschenken ins Trinkgläschen, in 3. Reihe unmittelbar nach dem Ausspeien. Die Differenzen sind natürlich grösser (durch Eintauchen des Thermometers etc.) als in Wirklichkeit bei den Versuchen, wie sich bei T 35 zeigt. Diese sollte durch das in Mund nehmen jedenfalls nicht kälter werden. Die 4. Reihe enthält die Anzahl der einzeinen Messungen, deren Mittel die betreffenden Angaben sind.

Tabelle d.

\begin{tabular}{ll|c|c|c|c|c|c|c|c|c|c|c} 
Reihe & 1 & 6 & 5,5 & 5 & 35 & 36 & 37,5 & 60 & 59 & 58 & 50 & 48 \\
Reihe 2 & 6,5 & 6,5 & 6 & 34,5 & 35,8 & 36 & 55 & 55 & 54 & 47 & 46 \\
Reihe 3 & 11 & 10,5 & 10,5 & 34 & 35 & 35,3 & 48 & 49 & 48,8 & 43,5 & 43.5 \\
Reihe 4 & 4 & 6 & 4 & 4 & 4 & 4 & 2 & 2 & 2 & 4 & 4
\end{tabular}

4. Folge der Lösungen.

Um den Einfluss der Folge der Lösungen festzustellen, wurden dieselben in 3 Kreise gestellt und jeder Kreis an einem Versuchstag nach beiden Richtungen hin durchgemacht. Die im Folgenden aufgezeichneten Entscheidungen enthalten demgemäss für jeden Kreis gleich viele Beiträge der einzelnen Temperaturen.

Tabelle e.

1. Kreis.

\begin{tabular}{|c|c|c|c|c|c|c|c|}
\hline orwärts & 2 & W & 6 & 3 & 5 & W & \\
\hline $\begin{array}{lll}\text { Urtheil } & \text { I } & 7 \\
\end{array}$ & $\begin{array}{ll}7 & 55 \\
8 & 71\end{array}$ & $\begin{array}{l}92 \\
90\end{array}$ & $\begin{array}{l}90 \\
92\end{array}$ & $\begin{array}{l}35 \\
40\end{array}$ & $\begin{array}{l}66 \\
84\end{array}$ & & $d$ \\
\hline ückwts. & $\bar{V}$ & 3 & 6 & W & 4 & $\overline{2}$ & der \\
\hline $\begin{array}{lll}\text { rtheil } & \text { I } & 84 \\
& \text { II } & 86\end{array}$ & $\begin{array}{ll}6 & 84\end{array}$ & 40 & & $\begin{array}{l}92 \\
91\end{array}$ & 72 & $\begin{array}{l}24 \\
18\end{array}$ & \\
\hline
\end{tabular}


Die Grenzen der Schmeckbarkeit von Chlornatrium in wässriger Lösung. 327

2. Kreis.

\begin{tabular}{|c|c|c|c|c|c|c|c|c|}
\hline Vorwärts & 4 & 6 & 2 & $W$ & 5 & W & 3 & \\
\hline theil I & 29 & 89 & 4 & 91 & 87 & 88 & 9 & \\
\hline theil II & 59 & 91 & 11 & 92 & 82 & 88 & 50 & \\
\hline Rückwärts & 3 & $\mathrm{~W}$ & 5 & $\mathrm{~W}$ & 2 & 6 & 4 & \\
\hline Urtheil II & & $\begin{array}{l}87 \\
88\end{array}$ & $\begin{array}{l}85 \\
86\end{array}$ & $\begin{array}{l}92 \\
92\end{array}$ & $\begin{array}{l}41 \\
10\end{array}$ & $\begin{array}{l}87 \\
92\end{array}$ & $\begin{array}{l}47 \\
66\end{array}$ & \\
\hline
\end{tabular}

3. Kreis.

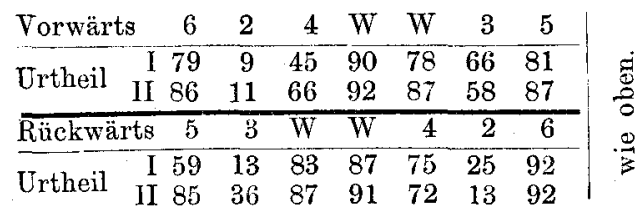

Es zeigt sich für I, weniger für II ein bedeutender Vorzug einmal der Lösungen, welche gegen das Ende einer Versuchsreihe hin geschmeckt wurden, den beim Beginn der Reihe gemachten Versuchen gegenüber; sodann der Lösungen, welche unmittelbar nach W. geschmeckt wurden denen gegenüber, welche stärkere Lösungen unmittelbar folgten. Von bew uster Erinnerung und Vergleichung ist hier durchaus keine Rede.

Erst später auf dieses Verhältniss aufmerksam, kann ich nur wenige Versuchsreihen paarweise gegenüberstellen, welche derselben Temperatur und demselben Kreis angehörig an einem Versuchstage zuerst, an einem andern zuletzt gemacht wurden. Der Einfluss einzelner Tage kann bei den wenigen Reihen stark stören.

Tabelle $\mathrm{f}$.

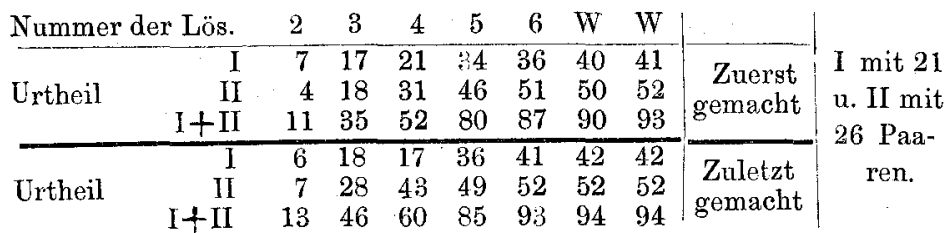

\begin{tabular}{|c|c|c|c|c|c|c|c|c|c|c|}
\hline \multicolumn{2}{|c|}{ Nummer der Lös. } & 2 & 3 & 4 & 5 & 6 & W & $W$ & & \multirow{6}{*}{$\begin{array}{l}\text { I mit } 25 \\
\text { u. II mit } \\
24 \text { Paa- } \\
\text { ren. }\end{array}$} \\
\hline \multirow{3}{*}{ Urtheil } & $I$ & 11 & 20 & 24 & 40 & 43 & 50 & 50 & \multirow{3}{*}{$\begin{array}{l}\text { Zweit } \\
\text { gemacht }\end{array}$} & \\
\hline & II & 8 & 19 & 31 & 45 & 46 & 48 & 47 & & \\
\hline & $I+I I$ & $\frac{19}{9}$ & $\frac{39}{18}$ & $\frac{55}{31}$ & $\frac{85}{43}$ & $\frac{89}{47}$ & $\frac{98}{48}$ & $\frac{97}{48}$ & & \\
\hline \multirow[t]{2}{*}{ Urtheil } & & 3 & 24 & 33 & 42 & 45 & 48 & 48 & letzt ge- & \\
\hline & $I+I I$ & 12 & 42 & 64 & 85 & 92 & 96 & 96 & macht. & \\
\hline
\end{tabular}

Bei den zuerst und zuletzt gemachten Versuchsreihen zeigt sich ein Vorzug zu Gunsten der letzten für II, beinahe kein Unter- 
schied für I; bei den zweiten und zweitletzten ein ganz geringer Vorzug zu Gunsten der zweitletzten.

Wenn ich das freilich unbedeutende Ergebniss der Morgen- und Abendversuche berücksichtigen darf, so fände es wohl seine Erklärung darin, dass die Morgenversuche (mit Ausnahme des Morgenkaffees) am betreffenden Tage die erstgemachten Geschmacksübungen gegenüber den Abendversuchen als auf die Uebung des gesammten Tages folgend, darstellen.

\section{Einige weitere Bemerkungen.}

Ich stelle im Folgenden die 100 mit Schneewasser gemachten Versuchsreihen den 100 Reihen mit Temperatur $10^{\circ}$ und $35^{\circ}$ gegenüber.

$$
\text { Tabelle } \mathrm{g} \text {. }
$$

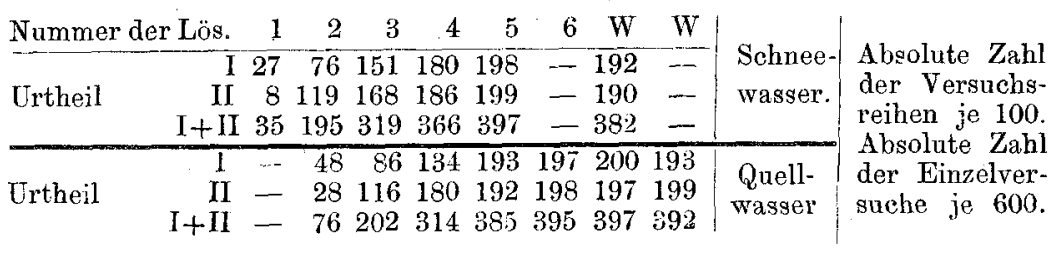

Der bedeutende Vorzug der Schneewasserversuche kann selbstverständlich weder der veränderten Empfindlichkeit, noch der veränderten Temperatur in erheblichem Maasse zugeschrieben werden.

Zur Vergleichung von I und If dient:

\section{Tabelle $\mathrm{h}$}

\begin{tabular}{|c|c|c|c|c|c|c|c|c|c|c|c|c|}
\hline \multirow{2}{*}{$\begin{array}{l}\text { Numme } \\
\text { Urtheil }\end{array}$} & ler Lös & 1 & 2 & 3 & 4 & 5 & 6 & $W$ & $W$ & & \multirow{2}{*}{\multicolumn{2}{|c|}{ 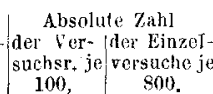 }} \\
\hline & II & $\begin{array}{r}27 \\
8\end{array}$ & $\begin{array}{r}76 \\
119\end{array}$ & $\begin{array}{l}151 \\
168\end{array}$ & $\begin{array}{l}180 \\
186\end{array}$ & $\begin{array}{l}198 \\
199\end{array}$ & - & $\begin{array}{l}192 \\
190\end{array}$ & 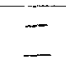 & $\begin{array}{l}\text { Schnee- } \\
\text { wasser. }\end{array}$ & & \\
\hline Urtheil & $\begin{array}{c}\text { I } \\
\text { II }\end{array}$ & - & $\begin{array}{r}100 \\
59\end{array}$ & $\begin{array}{l}192 \\
231\end{array}$ & $\begin{array}{l}295 \\
366\end{array}$ & $\begin{array}{l}415 \\
456\end{array}$ & $\begin{array}{l}466 \\
492\end{array}$ & $\begin{array}{l}493 \\
496\end{array}$ & $\begin{array}{l}479 \\
494\end{array}$ & $\begin{array}{l}\text { Quell- } \\
\text { wasser. }\end{array}$ & 250 & 1750 \\
\hline
\end{tabular}

Es zeigt sich, dass bei I die Zunahme der richtigen Urtheile langsamer geht, bei II aber zwischen der niedersten Nummer und der nächstfolgenden ein grosser Unterschied ist. I ist in der niedersten Nummer ziemlich besser als II, überall sonst schlechter. Es erklärt dieser ungleiche Gang der Zunahme Verschiedenheiten zwischen I und II (namentlich in der Tabelle e, weshalb dort I+II nicht berechnet ist); näher auf den Gang des Verlaufs werde ich erst auf Grundlage weiterer Erfahrungen eingehen.

Nachträglich sei über die Entscheidung ,unbestimmt" bemerkt, dass hier 2 Fälle zu unterscheiden sind: 1) man kann sich weder 
für "Salz" noch für „Wasser" entschieden aussprechen; 2) bei weitem in den meisten Fällen kann man mit Entschiedenheit "Wasser" ausschliessen, allein man schmeckt kein "Salz". Ueber diese minimalste Leistung des Sinnes dürften Versuche zugleich etwa mit reinem Wasser, Chlornatrium und einem bitteren Stoff Aufschluss geben.

Herrn Prof. von Vierordt, welcher mich zur Bearbeitung dieses Themas aufforderte und bei der Arbeit mit Rath und That unterstützte, sage ich hiefür meinen besten Dank.

\section{Bemerkungen \\ zu Preyers Abhandlung über die Grenzen des Empfindungsvermögens und Willens. \\ Von \\ v. Wittich.}

Preyers Abhandlung üher die Grenzen unseres Empfindens und Willens gab mir Veranlassung, eine Beobachtungsreihe wieder aufzunehmen, die ich bereits vor einigen Jahren begann, die ich aber, weil sie mir den damals gehofften Aufschluss über gewisse Fragen nicht gab, liegen liess, den Ausführungen P r e y e rs gegenüber, aber doch zum mindesten den Werth zu haben scheint, dass sie eine der Thatsachen, auf welche derselbe seine Betrachtungen stützt als irrthümlich hinstellt.

Bei Untersuchungen über die Fortpflanzungsgeschwindigkeit in menschlichen Nerven fand ich. wie ja auch vor mir Hirsch, Do nders und $\mathrm{Hanckel}$, dass die physiologische Zeit von Auge zu Hand ganz unverhältnissmässig länger ausfiel, als von Haut (Stirnhaut) zu Hand, dass demgemäss, die Zeit für die centralen Vorgänge in beiden Versuchsweisen als gleich angenommen, für die Opticusfasern eine sehr viel trägere Fortleitung zu berechnen kam, als für die Hautnerven. Mein erster Gedanke war, dass diese Verzögerung weniger in der Nervenbahn selbst zu finden sei, als vielmehr darin, dass bei den Augenversuchen der dem Opticus adae- 